\title{
Relationship between Serum Lipid Index, C Reaction Protein (CRP), Insulin Resistance and Coronary Artery Disease in Diabetes Mellitus Patients Complicated with Coronary Atherosclerotic Heart Disease (CAHD) and Correlation Analysis
}

\author{
Hui $\mathrm{Li}^{1}$, Zhanhua $\mathrm{Li}^{1,}{ }^{1}{ }^{,}$, Fanjin Zeng ${ }^{2}$, Shuhuai Guo ${ }^{1}$ \\ ${ }^{1}$ Xingtai Medial College, Xingtai, Hebei, 054000, China \\ ${ }^{2}$ Xingtai Polytechnic College, Xingtai, Hebei, 054000, China \\ a4099964@qq.com \\ ${ }^{*}$ Corresponding author
}

Keywords: Diabetes mellitus, Coronary heart disease, Coronary artery disease

\begin{abstract}
Objective: To investigate the relationship between serum lipid index, C Reaction protein (CRP), insulin resistance level and coronary artery disease (CAD) in patients with diabetes mellitus (DM) complicated with coronary atherosclerotic heart disease (CAHD) and its correlation. Method: Select 62 CAHD patients identified by coronary artery angiography in January 2015-January 2016 as DM group, 60 non-DM CAHD patients as NDM group, and 60 contemporary healthy people as control group, determine its blood glucose (GLU), insulin sensitivity index (ISI), level of insulin resistance (HOMA-IR), triglyceride (TG), total cholesterol (TC), high density lipoprotein cholesterol (HDL), low density lipoprotein cholesterol (LDL), and CRP level. Results: The incidence rate of multi-vessel in DM group is higher than that in NDM group $(\mathrm{P}<0.05)$; The levels of TC, TG, LDL, GLU, HOMA-IR and CRP in DM group are higher than that in NDM group and control group $(\mathrm{P}<0.05)$, while HDL and ISIS levels are lower $(\mathrm{P}<0.05)$; the levels of TC, TG, LDL, GLU, HOMA-IR and CRP in 3-vessel lesion group are significantly higher than that in 2-vessel lesion group and control group $(\mathrm{P}<0.05)$, while the levels of HDL and ISI are lower $(\mathrm{P}<0.05)$; By level correlation analysis, we found that the number of lesion artery is positively correlated with TC, TG, LDL, GLU, HOMA-IR, CRP levels $(\mathrm{P}<0.05)$, and negatively correlated with HAL, ISI level $(\mathrm{P}<0.05)$. Conclusion: Most CAHD patients exists severe lipid metabolism disorders, insulin resistance and inflammatory reaction, and its incidence level is related with the degree of coronary artery lesion, which may be the risk factors of coronary heart disease.
\end{abstract}

\section{Introduction}

Diabetes is a common clinical chronic metabolic disease. If the GLU is not controlled timely, with the progression of the disease, it's easy complicated with coronary atherosclerotic heart disease (CAHD), which is one of the main causes of death. In recent years, with the continues study on the pathogenesis of DM complicated with coronary heart disease, people found that its CAHD level is more serious than CHD patients, and the most common one is multi-vessel coronary artery change [1]. This paper measures the serum lipid index, IR and CRP, aims at explores its relationship with the degree of coronary artery disease. 


\section{Materials and Methods}

Clinical Materials. Select 62 CAHD patients in January 2015-January 2016 as DM group, all diagnosed by coronary artery angiography, coronary artery stenosis $\geq 50 \%$, with DM history, which consistent with WHO diagnostic criteria for diabetes [2]. There are 36 males, 26 females, age in 46-78 with an average age of (58.2 \pm 2.4$)$, among which, 14 patients have one lesion vessel, 32 have two lesion vessels and 16 have three lesion vessels. Select 60 CHD patients without DM as NDM group. There are 35 males and 25 females, age in 47-78 with an average age of (58.5 \pm 2.5 ), among which, 33 patients have one lesion vessel, 19 have two lesion vessels and 8 have three lesion vessels. Select 60 contemporary healthy people as control group. There are 36 males, 24 females, age in 45-78 with an average age of (57.8 \pm 2.2$)$. All the patients were not associated with autoimmune diseases, stroke, acute or chronic infectious diseases. The sex and age in the three groups are comparable $(P>0.05)$.

Method. First, draw venous blood $4 \sim 5 \mathrm{~mL}$, empty stomach in the morning, then, determine the GLU, TG, total cholesterol, HDL, LDL level of patients by American Beckman Coulter AU5800 automatic biochemical analyzer and level of serum insulin (FNS) was measured by electrochemical luminescence, reagents are supplied by Bayer company, the level of serum fasting blood glucose (FBG) was measured by glucose oxidase method, and the level of CRP was measured by radioimmunoassay.

Observation Index. Insulin resistance (IR) was evaluated according to the insulin sensitivity index (ISI) and the insulin resistance index (HOMA-IR). The formula is: HOMA-IR= FNS(mIU/L) $\times F B G(m m o l / L) / 22.5$ (conversion of units: Ipmll/L=1mIU/L $\times 6.965)$. The diagnosis of diabetes is based on WHO diabetes diagnosis and classification criteria [3].

According to the results of coronary artery angiography, the degree of coronary artery disease is measured [4], referring the right coronary artery, left anterior descending artery, left circumflex artery, the left main branch is divided into one, two or three lesions. Based on the diagnostic criteria for coronary heart disease in the American College of Cardiology (ACC) angiography guidelines, the lesion degree is determined by the maximum stenosis of each vessel, using semi-quantitative analysis, 1 score: stenosis degree $0 ; 2$ score: $1 \% \leq$ stenosis degree $\leq 25 \% ; 2$ score: $26 \% \leq$ stenosis degree $\leq 50 \%$; 3 score: $26 \% \leq$ stenosis degree $\leq 50 \% ; 4$ score: $76 \% \leq$ stenosis degree $\leq 99 \%$; 5 score: stenosis degree $100 \%$, measure the stenosis score of above three vascular. The coronary heart disease diagnostic criteria: at least one vessel's stenosis degree $\geq 50 \%$.

Statistical Analysis. The data were analyzed by SPSS19.0 statistical software. The $t$ test was used to compare the clinical data between groups, show as $(\bar{x} \pm s)$. The data was analyzed by $\chi^{2}$ test, to compare the degree of vascular lesion between groups, as n (\%). And the relationship of coronary artery disease lesion vessels and each indicators is using the level of correlation analysis to identify, hypothesize $P<0.05$, there exists statistical significance between groups.

\section{Result}

Degree of Coronary Artery Disease. The incidence rate of multi-vessel disease in DM group is significantly higher than that in NDM group $(P<0.05)$; but there was no statistical significant between the vascular stenosis score of two groups $(P>0.05)$, as show in Table 1 : 
Table 1 Ddegree analysis of coronary artery disease between DM group and NDM group

\begin{tabular}{|l|l|l|c|c|c|}
\hline Group & Case & 1-vessel lesion & 2-vessel lesion & 3-vessel lesion & $\begin{array}{l}\text { Lesion vascular score } \\
\text { (point) }\end{array}$ \\
\hline DM group & 62 & $14(22.58)$ & $32(51.61)$ & $16(25.81)$ & $7.45 \pm 0.65$ \\
\hline $\begin{array}{l}\text { NDM } \\
\text { group }\end{array}$ & 60 & $33(55.00)$ & $19(31.67)$ & $8(13.33)$ & $6.28 \pm 0.59$ \\
\hline$\chi^{2} / t$ & & 22.1337 & 8.1808 & 4.9476 & 1.2137 \\
\hline$P$ & & 0.0000 & 0.0042 & 0.0261 & 0.5811 \\
\hline
\end{tabular}

The Compare of Serum Lipid Index, HOMA-IR and CRP Level in Different Groups. In DM group, the level of TC, TG, LDL, GLU, HOMA-IR and CRP is higher than that in NDM group and control group $(P<0.05)$, and the level of HDL and ISI is lower $(P<0.05)$, as show in Table 2:

Table 2 Compare of serum lipid index, HOMA-IR and CRP level in different groups $(\bar{x} \pm s)$

\begin{tabular}{|l|l|l|l|l|l|l|l|l|l|}
\hline Group & $\begin{array}{l}\text { ca } \\
\text { se }\end{array}$ & $\begin{array}{l}\text { TC(mmo } \\
\text { l/L) }\end{array}$ & $\begin{array}{l}\text { TG(mm } \\
\text { ol/L) }\end{array}$ & $\begin{array}{l}\text { HDL(m } \\
\text { mol/L) }\end{array}$ & $\begin{array}{l}\text { LDL(mm } \\
\text { ol/L) }\end{array}$ & $\begin{array}{l}\text { GLU(mm } \\
\text { ol/L) }\end{array}$ & $\begin{array}{l}\text { ISI(mmol } \\
/ \mathrm{L})\end{array}$ & $\begin{array}{l}\text { HOMA-I } \\
\text { R(mmol/ } \\
\text { L) }\end{array}$ & $\begin{array}{l}\text { CRP( } \\
\mathrm{mg} / \mathrm{L})\end{array}$ \\
\hline $\begin{array}{l}\text { DM } \\
\text { group }\end{array}$ & 62 & $\begin{array}{l}4.26 \pm 1.1 \\
8 * \#\end{array}$ & $\begin{array}{l}4.52 \pm 1 . \\
23^{*} \#\end{array}$ & $\begin{array}{l}0.86 \pm 0.1 \\
2 * \#\end{array}$ & $\begin{array}{l}3.89 \pm 1.0 \\
4 * \#\end{array}$ & $\begin{array}{l}11.76 \pm 2.8 \\
6 * \#\end{array}$ & $\begin{array}{l}1.72 \pm 0.16 \\
* \#\end{array}$ & $\begin{array}{l}3.27 \pm 0.86 \\
* \#\end{array}$ & $\begin{array}{l}9.26 \pm \\
1.54^{*} \\
\#\end{array}$ \\
\hline $\begin{array}{l}\text { NDM } \\
\text { group }\end{array}$ & 60 & $\begin{array}{l}2.54 \pm 0.8 \\
2\end{array}$ & $\begin{array}{l}2.68 \pm 0 . \\
89\end{array}$ & $\begin{array}{l}1.26 \pm 0.2 \\
1\end{array}$ & $\begin{array}{l}2.76 \pm 0.6 \\
2\end{array}$ & $7.18 \pm 2.01$ & $2.21 \pm 0.25$ & $2.01 \pm 0.46$ & $\begin{array}{l}5.37 \pm \\
0.76\end{array}$ \\
\hline $\begin{array}{l}\text { Contr } \\
\text { ol } \\
\text { group }\end{array}$ & 60 & $\begin{array}{l}1.32 \pm 0.3 \\
5\end{array}$ & $\begin{array}{l}1.36 \pm 0 . \\
37\end{array}$ & $\begin{array}{l}1.85 \pm 0.3 \\
2\end{array}$ & $\begin{array}{l}2.23 \pm 0.3 \\
4\end{array}$ & $4.17 \pm 1.16$ & $2.89 \pm 0.43$ & $0.16 \pm 0.12$ & $\begin{array}{l}1.76 \pm \\
0.34\end{array}$ \\
\hline
\end{tabular}

Note: compare with NDM group, ${ }^{*} P<0.05$; compare with control group, $\# P<0.05$.

Comparison of Serum Lipid Index, HOM-IR and CRP Level in Different Vascular Disease Groups. In 3-vessel lesion group, the levels of TC, TG, LDL, GLU, HOMA-IR and CRP are higher than2-vessel lesion group and control group $(P<0.05)$, and its HDL and ISI level are lower than them $(P<0.05)$, show in Table 3:

Table 3 Comparison of serum lipid index, HOM-IR and CRP level in different vascular disease groups $(\bar{x} \pm s)$

\begin{tabular}{|c|c|c|c|c|c|c|c|c|}
\hline Group & $\begin{array}{l}\text { TC(mm } \\
\mathrm{ol} / \mathrm{L})\end{array}$ & $\begin{array}{l}\text { TG(mmo } \\
\text { l/L) }\end{array}$ & $\begin{array}{l}\mathrm{HDL}(\mathrm{mm} \\
\mathrm{ol} / \mathrm{L})\end{array}$ & $\begin{array}{l}\text { LDL(mmo } \\
\text { l/L) }\end{array}$ & $\begin{array}{l}\text { GLU(mmo } \\
\text { l/L) }\end{array}$ & $\begin{array}{l}\text { ISI(mmol } \\
\text { /L) }\end{array}$ & $\begin{array}{l}\text { HOMA-IR } \\
(\mathrm{mmol} / \mathrm{L})\end{array}$ & $\begin{array}{l}\text { CRP(mg/ } \\
\mathrm{L})\end{array}$ \\
\hline $\begin{array}{l}\text { 3-vesse } \\
1 \\
\text { lesions }\end{array}$ & $\begin{array}{r}5.12 \\
\pm 1.43 * \#\end{array}$ & $\begin{array}{l}5.28 \pm \\
1.45^{* \#}\end{array}$ & $\begin{array}{l}0.82 \pm \\
0.13^{*} \#\end{array}$ & $\begin{array}{l}4.05 \pm 1 \\
.23^{*} \#\end{array}$ & $\begin{array}{l}9.45 \pm 2 . \\
65^{* \#}\end{array}$ & $\begin{array}{l}1.32 \pm \\
0.14^{*} \#\end{array}$ & $\begin{array}{l}3.56 \pm 0 . \\
75^{* \#}\end{array}$ & $\begin{array}{r}10.36 \\
\pm 1.48^{* \#}\end{array}$ \\
\hline $\begin{array}{l}\text { 2-vesse } \\
1 \\
\text { lesions }\end{array}$ & $\begin{array}{r}2.86 \\
\pm 1.02\end{array}$ & $\begin{array}{l}3.27 \pm \\
1.19\end{array}$ & $\begin{array}{l}1.29 \pm \\
0.22\end{array}$ & $.68^{3.26 \pm 0}$ & $85^{6.32 \pm 1 .}$ & $\begin{array}{l}2.27 \pm \\
0.24\end{array}$ & $48^{2.14 \pm 0 .}$ & $\begin{array}{l}6.23 \pm \\
0.75\end{array}$ \\
\hline $\begin{array}{l}\text { Control } \\
\text { group }\end{array}$ & $\begin{aligned} & 1.32 \\
& \pm 0.35\end{aligned}$ & $\begin{array}{l}1.36 \pm \\
0.37\end{array}$ & $\begin{array}{l}1.85 \pm \\
0.32\end{array}$ & $.34^{2.54 \pm 0}$ & $16^{4.17 \pm 1 \text {. }}$ & $\begin{array}{l}2.89 \pm \\
0.43\end{array}$ & $12^{0.16 \pm 0 .}$ & $\begin{array}{l}1.76 \pm \\
0.34\end{array}$ \\
\hline
\end{tabular}

Note: compare with two lesion branches, $* P<0.05$; compare with control group, $\# P<0.05$. 
Correlative Analysis between the Number of Coronary Lesion Artery and Serum Lipid Index, HOMA-IR and CRP Level. Based on level correlation analysis, the number of affection artery is positively correlated with TC, TG, LDL, GLU, HOMA-IR, CRP levels $(P<0.05)$, and negatively correlated with HAL, ISI level $(P<0.05)$, as shown in table 4:

Table 4 Correlative analysis between the number of coronary lesion artery and serum lipid index, HOMA-IR and CRP level

\begin{tabular}{|l|l|l|}
\hline Index & $r$ & $P$ \\
\hline TC & 0.24 & 0.02 \\
\hline TG & 0.22 & 0.01 \\
\hline HDL & -1.22 & 0.04 \\
\hline LDL & 0.23 & 0.04 \\
\hline GLU & 0.19 & 0.01 \\
\hline ISI & -0.62 & 0.03 \\
\hline HOMA-IR & 0.33 & 0.03 \\
\hline CRP & 0.25 & 0.02 \\
\hline
\end{tabular}

\section{Discussion}

In recent years, with the changes of people's lifestyle, the incidence rate of diabetes has an obvious upward trend, resulting the number of CAHD patients increased gradually. Diabetes mellitus is an important motivation of coronary heart disease, if not treated timely, it can easily lead to heart failure, stroke and other diseases with a high mortality rate. Studies have reported [5], the mortality rate of type 1 diabetes mellitus complicated with CAHD patients is 10-14 times higher than pure coronary heart disease and the mortality rate of type 2 is 2-4 times higher. For the occurrence of CAHD disease, at present, the regulate of abnormal glucose metabolism is the clinical target, the method includes regulate blood lipid, reduce blood pressure, quit smoking and alcohol, but its morbidity and mortality are still higher than NDM group and should pay more attention to.

Analysis the mechanism of CAHD, in recent years, studies have confirmed [6], IR and hyperglycemia can reduce the generation of nitric and oxide which derived by DM endothelial, thereby causing vascular endothelial damage; at the same time, most DM patients exists Calcium ion disorder phenomenon, the significance increased glucose concentration in platelets, coupled with the activation of large number of protein kinase $\mathrm{C}$, reduced the production of $\mathrm{N}$ and $\mathrm{O}$, damaging the platelet and made the coagulation function abnormal. Therefore, the activation and aggregation of platelets in vivo, together with coagulation dysfunction is associated with the forming of thrombus after the coronary atherosclerotic plaque rupture. This study shows that, the GLU level in DM group is higher than NDM group and control group $(P<0.05)$, which was similar to that reported in literature [7]. A large number of studies have confirmed [8], coronary atherosclerosis and thrombosis is the main cause of coronary heart disease, and the lipid metabolism disorders in vivo can accelerate the occurrence of atherosclerosis. Studies have reported [9], the level of HDL and coronary artery disease was negatively correlated, and that is with each $0.05 \mathrm{mmol} / \mathrm{L}$ drop of HDL level in patients, the risk of coronary heart disease increased by $4 \%-5 \%$. In this study, we found that the level of HDL in DM group is significantly lower than that in NDM group and control group. It shows that strengthen the monitoring on blood lipid levels in diabetes patients plays an important role in prevention the occurrence of cardiovascular disease. Studies have shown that [10], the inflammation reaction is one of the risk factors for coronary atherosclerosis 
heart disease. The vascular endothelial injury can cause fibrosis and a series of inflammation reactions, which will lead to a large number of foam cells infiltrating the plaque fiber cap, further resulting atherosclerotic plaque rupture. CRP is a kind of response protein which is expressed by hepatocytes when it is damaged like injury or envisioned by microorganisms. It is a sensitive index of inflammatory reaction and can be used to monitoring the occurrence and development of cardiovascular disease at present [11]. This study found that, the CRP level in DM group is higher than that in NDM group and control group $(P<0.05)$, similar to those reported in literature, indicating that the occurrence of CAHD is related with response protein in acute phase. Meanwhile, the level of HOMA-IR in DM is obviously higher than that in NDM group and control group and ISI level is lower than them $(P<0.05)$. The main reason is that CRP is one of the risk motivations of coronary heart disease, which plays an importance role in the formation and development of atherosclerosis, and its increase will promote the IR level which further promotes the formation and development of atherosclerosis [12-13]. For diabetes patients, the common pathological features are pancreatic islet cell dysfunction and insulin resistance. IR and decreased insulin sensitivity can cause lipid metabolism disorders, accelerate the decomposition of body fat by inhibiting the conversion of glucose in vivo, thereby promoting the increase of synthesis of TC, TG and LDL. This study shows that the TC, TG and LDL level in DM group are higher than that in NDM group and control group $(P<0.05)$, similar with the results in Sun Yuqin's report [14].

Analysis the relationship between the degree of coronary artery disease and blood lipid metabolism, insulin resistance and inflammatory reaction, it was found that most diabetic patients showed right coronary artery, left anterior descending artery and left circumflex artery lesions, meanwhile, with the increase of TC, TG, LDL and CRP levels, the number of lesion artery increased either, while HDL is on the opposite. This study found that, the levels of TC, TG, LDL, GLU, HOMA-IR and CRP in 3-vessel lesion group are significantly higher than that in 2-vessel lesion group and the control group $(P<0.05)$, similar with Lin Zhuandi's report [15]. For one lesion vessel group, duo to its limited lesion circumscription, the percutaneous coronary intervention treatment can achieve a good effect. Majority of 2-vessel lesion and 3-vessel lesion patients have coronary artery diffuse stenosis and the effect of intervention therapy is poor, therefore coronary artery bypass grafting is needed. Through correlation analysis, this paper found the number of lesion vessels is positively related with the level of TC, TG, LDL, GLU, HOMA-IR and CRP in diabetic patients $(P<0.05)$, and was negatively correlated with HDL AND ISI levels $(P<0.05)$, which is similar to the report in literature, indicating that the above indicators can effectively predicting the extent of coronary artery disease in diabetic patients.

In summary, in the process of DM patients predicting complicated coronary heart disease, the lipid metabolism index, insulin resistance and CRP inflammatory response indicators plays an importance role in reflecting its lesion degree, which can be used as a predictive factor for clinical prevention of cardiovascular disease, it has a good application value in clinic.

\section{Acknowledgement}

This paper is supported by Xingtai Science and Technology Support Project, China (No. 2012ZC049).

\section{References}

[1] Ren W, Guo L. Analysis on Risk Factors and Coronary Artery Features in Patients with Type 2 Diabetes Complicated with Coronary Heart Disease. Shanxi Medical Journal, 2014, 9 (16), 


\section{p.p. 1896-1898. (In Chinese)}

[2] Zhang J, Jiang Z. Clinical Value of Retinol-Binding Protein and Urinary Albumin in the Diagnosis of Coronary Artery Disease in Patients with Diabetes Mellitus and Coronary Heart Disease. Medical Recapitulate, 2015, 21 (22), p.p. 4152-4154. (In Chinese)

[3] Li H, LYU H, Lu J, et al. Correlative Analysis of Coronary Artery Lesion Degree with Glycosylated Hemoglobin and Bilirubin Levels in Patients with Diabetes Mellitus and Coronary Atherosclerotic Heart Disease. Journal of Shanghai Jiaotong University (Medical Science), 2016, 36 (2), p.p. 233-236, 242. (In Chinese)

[4] Zhang C, Zheng Z, Mu N, et al. Relationship between Carotid Artery Ultrasonography and Coronary Artery Disease in Diabetes Patients with Coronary Heart Disease. Practical Journal of Cardiac Pneumal and Vascular Disease, 2009, 17 (4), p.p. 243-245. (In Chinese)

[5] Saely, C.H., Drexel,H. Is Type 2 Diabetes Really a Coronary Heart Disease Risk Equivalent? Vascular Pharmacology, 2013, 59 (1/2), p.p.11-18.

[6] Wei Q, Jin H, Jiang Y, et al. Correlation between Plasma Osteopontin and Severity of Coronary Artery Disease in Non-diabetes Patients with Coronary Heart Disease. Chinese Journal of Arteriosclerosis, 2014, 22 (5), p.p. 477-479. (In Chinese)

[7] Sun Y, Dong S, Ren Z, et al. Study on Features of Coronary Artery Lesion and Patients in Type 2 Diabetes Mellitus Complicated with Coronary Heart Disease. Chinese Journal of Cardiovascular Rehabilitation, 2014, 23 (1), p.p. 33-36. (In Chinese)

[8] Wu F. Characteristics of Coronary Artery Disease in Diabetic Patients with Coronary Heart Disease. China Foreign Medical Treatment, 2011, 30 (30), p.p. 42. (In Chinese)

[9] Wang D, Feng X, Zhou Y, et al. Study on the Relationship between Diabetes Mellitus Complicated with Coronary Heart Disease and Features of Coronary Artery Disease. Chinese Circulation Journal, 2015, 23 (1), p.p. 60. (In Chinese)

[10] Chen F, Xiao C. Correlation analysis between serum inflammatory cytokines and Coronary Artery Lesions in Patients with Type 2 Diabetes Mellitus. Journal of Chinese Physician, 2010, 12 (1), p.p. 92-95. (In Chinese)

[11] Sun Y, Tong Y. Analysis of Coronary Artery Lesions in Patients with Coronary Heart Disease Complicated with Type 2 Diabetes Mellitus. China Medical Herald, 2010, 7 (6), p.p. 54-55. (In Chinese)

[12] $\mathrm{Hu} \mathrm{S}$, He W, Liu Z,W et al. The Accumulation of Glycoxidation Product N-(epsilon)-Carboxymethyllysine in Cardiac Tissues with Age, Diabetes Mellitus and Coronary Heart Disease. The Tohoku Journal of Experimental Medicine, 2013, 230(1), p.p. 25-32.

[13] Wu W, Sun Z, Li Q. et al. Influence of the Glucose-Lowering Rate on Left Ventricular Function in Patients with Type 2 Diabetes and Coronary Heart Disease. Journal of Diabetes and Its Complications, 2012, 26(2):83-88.

[14]Sun Y, Han H, Song W, et al. Correlative Study of Lipid Metabolism and Coronary Artery Lesions in Coronary Heart Disease Patients Complicated with Type 2 Diabetes Mellitus. Chinese Journal of Laboratory Diagnosis, 2010, 14(11), p.p. 1788-1790. (In Chinese)

[15] Lin Z. Correlative Study between Coronary Heart Disease and Diabetes Mellitus Complicated with Coronary Artery Disease. The Journal of Practical Medicine, 2009, 25 (13), p.p. 2097-2099. (In Chinese) 